\title{
Energy Efficiency Analysis of Link Layer Backoff Schemes on Point-to-Point Markov Fading Links
}

\author{
P. M. Soni and A. Chockalingam \\ Wireless Research Lab \\ Department of Electrical Communication Engineering \\ Indian Institute of Science, Bangalore 560012, INDIA \\ fax: +91-80-360-0563-e-mail: \{sonipm, achockal\}@ece.iisc.ernet.in
}

\begin{abstract}
Backoff algorithms are traditionally employed in multiple access networks, like Ethernet, to recover from packet collisions. In this paper, we propose and carry out the analysis for three types of link layer backoff schemes-linear, binary exponential, geometric-on point-to-point wireless fading links, where packet errors occur non-independently. In such a scenario, the backoff schemes are shown to achieve better energy efficiency without compromising much on the link layer throughput performance.
\end{abstract}

Keywords - Backoff algorithms, fading channels, energy efficiency.

\section{Introduction}

Backoff algorithms are typically used in multiple access networks to recover from packet collisions. For example, a truncated binary exponential backoff scheme is employed in Ethernet [1]. The backoff delay is increased by larger and larger amounts on each successive collision, up to a finite number of retransmission attempts. Here we propose that backoff schemes could be applied beneficially on point-to-point wireless links as well. The motivation arises from the potential for substantial energy savings when the link experiences deep fades and bursty errors.

During channel fades, it is likely that a large number of consecutive packets are received in error due to memory in the multipath fading process [2]. A backoff scheme at the link layer (LL), applying an appropriate backoff rule upon each LL packet error event, can hold the channel idle for some specified number of slots, thereby reducing the energy spent in wastefully sending a big chunk of possible failed packet transmissions. This is done at a cost of possible reduction in the throughput. But, in mobile

0-7803-6465-5/00 \$10.00@ 2000 IEEE stations, saving battery power can outweigh a slight loss in throughput [3].

Nonindependent errors on wireless channels are, with reasonable accuracy, modeled by a first-order Markov chain in most analyses in literature [4],[5]. Link layer ARQ protocols, like Go-Back-N and Selective Repeat, have been analyzed in the presence of nonindependent errors in [6]-[10]. However, all these studies focus only on throughput and delay performance and do not consider energy savings through LL backoff strategies. Our new contribution in this letter is the proposal and throughput-energy efficiency analysis of three easily implementable LL backoff strategies, namely, linear backoff (LBO), binary exponential backoff (BEBO), and geometric backoff (GBO). We use the Markov chain representation of the wireless channel with Markov parameters $p$ and $(1-q)$ being the probabilities that the $i^{\text {th }}$ packet transmitted is in success given the $(i-1)^{t h}$ packet was successful and unsuccessful, respectively. Numerical results show that the proposed backoff schemes achieve better energy efficiency without compromising much on the link layer throughput performance.

\section{LL Backoff Algorithms}

Among the LL ARQ protocols, Selective Repeat (SR) gives the best throughput performance. The maximum throughput achieved by ideal SR scheme is bounded by $(1-\epsilon)$ where $\epsilon$ is the average packet error rate on the link [8]. Here we present and analyze three different link layer backoff schemes - linear, binary exponential, and geometric backoffs - which are defined as follows.

Linear Backoff: In a linear backoff scheme, on $i^{\text {th }}$ successive fail of a packet, the LL keeps idle for $i$ number of subsequent slots, i.e., the backoff delay grows linearly on each successive fail. 
Binary Exponential Backoff: In this scheme, the LL keeps idle for $2^{i}-1$ number of slots on $i^{\text {th }}$ successive fail.

Geometric Backoff: In this scheme, there is a parameter $g, 0<g \leq 1$. Following an idle or packet fail, the LL keeps idle in the next slot with probability $g$ (or equivalently, transmits a packet with probability $1-g$ ). In other words, the expected number of backoff slots following a fail is given by $g /(1-g)$.

\section{Analysis}

We are interested in calculating the throughput and energy efficiency of the above backoff schemes and comparing them with the performance of ideal SR scheme without backoff. We assume instantaneous and perfect feedback in our analysis. The detailed mathematical analysis goes as follows.

Define $\delta_{i}$ and $\gamma_{i}$ as the probabilities that success and fail occur, respectively, at $(k+i)^{t h}$ slot given the $k^{t h}$ slot is a fail for any $k \geq 1$ and $i \geq 1$. Let 1 represent a fail, 0 represent a success and ' $x$ ' represent either of them, and let $P\{A\}$ denote the probability of event $A$. Then for $i \geq 1$,

$$
\begin{aligned}
& \delta_{i}=P\{0 \overbrace{x x \cdots x x}^{i-1} \mid 1\} \\
& \gamma_{i}=P\{1 \overbrace{x x \cdots x x}^{i-1} \mid 1\}
\end{aligned}
$$

Observe that, for any $i \geq 1, \delta_{i}+\gamma_{i}=1$. With boundary conditions $\delta_{1}=1-q$ and $\gamma_{1}=q$, we can write recursive relations on $\delta_{i}$ and $\gamma_{i}$ as,

$$
\begin{aligned}
& \delta_{i}=\gamma_{i-1}(1-q)+\delta_{i-1} p, i \geq 2 \\
& \gamma_{i}=\gamma_{i-1} q+\delta_{i-1}(1-p), i \geq 2
\end{aligned}
$$

Assume that the first packet was transmitted at time $t=t_{0}$. We have two cases corresponding to the first packet transmitted being a success or a fail. Let $\tau$ be the packet duration, assumed constant. Then a cycle is defined as follows.

Case (a): First packet transmitted at $t=t_{0}$ is a success. Let the first fail occur at $t=t_{0}+n \tau$, i.e., first $n$ packets are in success. Since the $(n+1)^{t h}$ packet is a fail, the next $D_{1}$ slots are kept idle and a packet is transmitted again at $t=t_{0}+(n+1+$ $\left.D_{1}\right) \tau$. If this packet again fails, next $D_{2}$ number of slots are kept idle and a packet is transmitted at $t=t_{0}+\left(n+1+D_{1}+1+D_{2}\right) \tau$, and so on. Finally a packet gets through, say, after $l$ number of attempts (i.e., $l$ fails) which was transmitted at $t=t_{0}+\left[n+l+\sum_{i=1}^{l} D_{i}\right] \tau=t_{1}$. Since the packet trans mitted at $t_{1}$ is a success and we are assuming a firstorder Markov model, the future evolution of the successfail-idle sequence is same as that started at $t_{0}$. Notice that, for linear backoff, $D_{i}=i$ and for binary exponential backoff, $D_{i}=2^{i}-1$. The duration $t_{1}-t_{0}$ is defined as the first cycle. In general, $t_{i}$ is defined as the epoch at which the $(i+1)^{t h}$ cycle begins (see Figure 1).

Case(b): First packet transmitted at $t=t_{0}$ is a fail. In this case, the transmitter keeps idle for next $D_{1}$ slots and makes an attempt at the $\left(D_{1}+1\right)^{t h}$ slot. Observe that the evolution of the sequence in this case is identical to the evolution in Case (a) that started at $t=t_{0}+n \tau$.

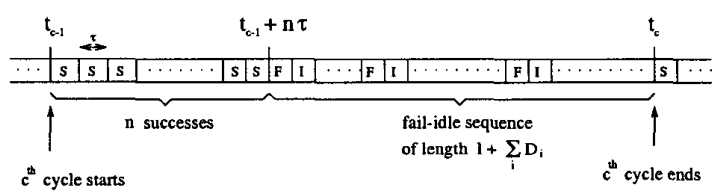

Figure 1: A transmission cycle in LL backoff schemes

Now define $T_{c}=t_{c}-t_{c-1}, c \geq 1$, as the duration of the $c^{t h}$ cycle. Since $\tau$ is a constant, we normalize $T_{c}$ with $\tau$ (i.e., $T_{c}$ is the number of slots in a cycle). $\mathrm{Ob}-$ serve that $\left\{T_{c}, c \geq 1\right\}$ is Markov renewal process. If the first packet is a success, then $\left\{T_{c}, c \geq 1\right\}$ are independent and identically distributed (i.i.d) random variables. But, If the first packet is a fail, we have $\left\{T_{c}, c \geq 2\right\}$ i.i.d random variables. $T_{1}$ will have a different distribution, but this does not prevent us from applying the Renewal Reward Theorem [11] which we use later in this section.

Let $S_{c}, F_{c}$ and $I_{c}$ be the random variables representing the number of successes, fails and idles in $c^{\text {th }}$ cycle, respectively. Let $f(k)$ be the probability that the length of an 'effective fade' is $k$, where the effective fade in a cycle is the total duration of the failidle sequence in that cycle. Let $n_{c}$ be the duration of a cycle, then for $\mathrm{BEBO}$ and $\mathrm{LBO}$, the minimum value $n_{c}$ can take is 3 , because a cycle should consist of at least one success, one fail and one idle. Write $k=n_{f}+n_{i}$, where $n_{f}$ and $n_{i}$ are the number of fails and idles, respectively, in that cycle. Observe that, in BEBO and LBO schemes, we have constraint on $n_{f}$, say $1 \leq n_{f} \leq r$, where $r$ is a number dependent on $n_{c}$. Also $n_{i}$ is dependent on $n_{f}$, and $n_{s}$ is dependent on both $n_{c}$ and $n_{f}$. In other words, for a given cycle length $n_{c}$, if the number of fails $n_{f}$ is fixed, $n_{i}$ and $n_{s}$ are also fixed. Now, the expected renewal life time, in number of slots, is given by,

$$
E\left[T_{c}\right]=\sum_{n_{c}=3}^{\infty} n_{c} \sum_{n_{f}=1}^{r} p^{\left(n_{s}-1\right)}(1-p) f\left(n_{f}+n_{i}\right) .
$$

Similarly,

$$
E\left[S_{c}\right]=\sum_{n_{c}=3}^{\infty} \sum_{n_{f}=1}^{r} n_{s} p^{\left(n_{s}-1\right)}(1-p) f\left(n_{f}+n_{i}\right),
$$




$$
\begin{aligned}
& E\left[F_{c}\right]=\sum_{n_{c}=3}^{\infty} \sum_{n_{f}=1}^{r} n_{f} p^{\left(n_{s}-1\right)}(1-p) f\left(n_{f}+n_{i}\right), \\
& E\left[I_{c}\right]=\sum_{n_{c}=3}^{\infty} \sum_{n_{f}=1}^{r} n_{i} p^{\left(n_{s}-1\right)}(1-p) f\left(n_{f}+n_{i}\right) .
\end{aligned}
$$

Let $\pi_{s}, \pi_{f}$ and $\pi_{i}$ be the stationary probabilities of success, fail and idle, respectively. We calculate these stationary probabilities as a reward rate of the above renewal process. Take the reward as the total duration of the fail slots. Then, $\tau F_{c}$ is the reward in the $c^{t h}$ cycle. The expected reward accrued till time $t, c(t)$, is the expected duration of fail slots in $[0, t]$. Now, applying Renewal Reward Theorem [11],

$$
\lim _{t \rightarrow \infty} \frac{c(t)}{t}=\frac{\tau E\left[F_{c}\right]}{\tau E\left[T_{c}\right]}=\frac{E\left[F_{c}\right]}{E\left[T_{c}\right]}, c \geq 2 .
$$

The LHS of the above equation gives the stationary probability of fail. In other words, $\pi_{f}=E\left[F_{c}\right] / E\left[T_{c}\right]$. Similarly, by taking the total success duration and idle duration as the reward, we get the stationary probabilities of success and idle, respectively as, $\pi_{s}=$ $E\left[S_{c}\right] / E\left[T_{c}\right]$ and $\pi_{i}=E\left[I_{c}\right] / E\left[T_{c}\right]$.

\subsection{Linear Backoff}

In linear backoff, we have $n_{i}=\frac{n_{f}\left(n_{f}+1\right)}{2}$ and $n_{s}=$ $\left(n_{c}-\frac{n_{f}\left(n_{f}+3\right)}{2}\right)$. Since there should be at least one success in a cycle we have the constraint, $\left(n_{c}-\frac{n_{f}\left(n_{f}+3\right)}{2}\right)>0$, which gives $n_{f} \leq r$ where, $r=\left\lceil\frac{\sqrt{9+8 n_{c}}-3}{2}\right\rceil-1$. Then,

$f\left(\frac{n_{f}\left(n_{f}+3\right)}{2}\right)= \begin{cases}\delta_{2} & : n_{f}=1 \\ \delta_{n_{f}+1} \prod_{m=2}^{n_{f}} \gamma_{m} & : 1<n_{f} \leq r\end{cases}$

We can obtain the stationary probabilities $\pi_{s}, \pi_{f}$ and $\pi_{i}$ by using these values of $n_{s}, n_{i}, r$ and $f(\cdot)$ in equations (5)-(8).

\subsection{Binary Exponential Backoff}

In this backoff scheme, the number of idle slots in a cycle equals $n_{i}=2\left(2^{n_{f}}-1\right)-n_{f}$ and the number of successes equals $n_{s}=n_{c}-2\left(2^{n_{f}}-1\right)$. Using the same arguments as in the linear backoff, we have the constraint, $n_{c}-2\left(2^{n_{f}}-1\right)>0$, which gives $n_{f} \leq r$, where, $r=\left\lceil\log \left(\frac{n_{c}}{2}+1\right)\right\rceil-1$ Then,

$f\left(2\left(2^{n_{f}}-1\right)\right)= \begin{cases}\delta_{2} & : n_{f}=1 \\ \delta_{2^{n} f} \prod_{m=1}^{n_{f}-1} \gamma_{2^{m}} & : 1<n_{f} \leq r\end{cases}$

Again, using these values of $n_{s}, n_{i}, r$ and $f(\cdot)$ in the equations (5)-(8), we get the stationary probabilities $\pi_{s}, \pi_{f}$ and $\pi_{i}$.

Truncated BEBO:
A variant of $\mathrm{BEBO}$, namely, truncated binary exponential backoff (T-BEBO), is also of interest. In T$\mathrm{BEBO}$, we have a parameter $m>0$. T-BEBO functions exactly same as the BEBO till $m$ consecutive fails, after which the transmitter gives up the backoff and continues with the transmission of the next LL packet. The analysis for the T-BEBO is similar to BEBO except that the cases in which the number of fails is less than or equal to $m$ and greater than $m$ need to be considered separately. When the number of fails is greater than $m$ (say, $m+k, k>0$ ), the first $m$ of them will be followed by a certain number (according to the BEBO rule) of idle slots and the remaining $k$ of them will be consecutive. Then, we can write $n_{c}-2\left(2^{m}-1\right)-k>0$ which gives $k \leq n_{c}-2^{m+1}+1$. Thus $r$ takes a value which is maximum of $\left(m+n_{c}-2^{m+1}+1\right)$ and $\left\lceil\log _{2}\left(\frac{n_{c}}{2}+1\right)\right\rceil-1$. When $n_{f}$ is less than $m, n_{s}$ and $n_{i}$ takes same values as that for BEBO without truncation, but when $n_{f}$ is greater than $m$, we get, $n_{i}=2\left(2^{m}-1\right)-m$ and $n_{s}=n_{c}-n_{f}-n_{i}$. With these, the expected value of $T_{c}$ can be written as,

$$
\begin{aligned}
E\left[T_{c}\right]= & \sum_{n_{c}=3}^{\infty} \sum_{n_{f}=1}^{r} n_{c} p^{n_{s}-1}(1-p)\left(I_{\left\{n_{f} \leq m\right\}} .\right. \\
& f\left(2\left(2^{n_{f}}-1\right)\right)+I_{\left\{n_{f}>m\right\}} \alpha(m) \\
& \left.q^{n_{f}-m-1}(1-q)\right)
\end{aligned}
$$

where, $\alpha(m)=\prod_{l=1}^{m} \gamma_{2^{\prime}}$ and $I_{\{A\}}$ is the indicator function which is 1 when the event $A$ happens and 0 otherwise. $E\left[S_{c}\right], E\left[F_{c}\right]$ and $E\left[I_{c}\right]$ for T-BEBO can be obtained by replacing $n_{c}$ by $n_{s}, n_{f}$ and $n_{i}$, respectively, in equation (10). Note that $m=\infty$ corresponds to BEBO without truncation.

\subsection{Geometric Backoff}

The approach we adopt to analyze the geometric backoff is as follows. Due to the memoryless property of the geometric backoff delay, the system can be modeled as a finite state Markov chain with the system status in a slot being in any one of success, fail, idle with possible success and idle with possible fail. Denote these states by $s, f, i_{s}$ and $i_{f}$, and the stationary probabilities by $\pi_{s}, \pi_{f}, \pi_{i_{s}}$ and $\pi_{i_{f}}$ respectively. Then, for the Markov chain, the stationary probability vector $\underline{\pi}=\left[\begin{array}{llll}\pi_{s} & \pi_{f} & \pi_{i_{s}} & \pi_{i_{f}}\end{array}\right]$, and the transition probability matrix $\mathbf{P}$ are related by $\underline{\pi}=\underline{\pi} \mathbf{P}$, where,

$$
\mathbf{P}=\left[\begin{array}{llll}
p & \bar{p} & 0 & 0 \\
\bar{g} \bar{q} & \bar{g} q & g \bar{q} & g q \\
\bar{g} p & \bar{g} \bar{p} & g p & g \bar{p} \\
\bar{g} \bar{q} & \bar{g} q & g \bar{q} & g q
\end{array}\right]
$$

where, $\bar{p}=(1-p), \bar{q}=(1-q)$, and $\bar{g}=(1-g)$. Solving for $\underline{\pi}=\underline{\pi} \mathbf{P}$, we get,

$$
\pi_{s}=\frac{(1-g)(1-q)}{(2-p-q)(1-g p)}
$$




$$
\pi_{f}=\frac{(1-p)}{(2-p-q)(1-g p)} \cdot M,
$$

and $\pi_{i}=\pi_{i_{s}}+\pi_{i_{f}}=1-\pi_{s}-\pi_{f}$, where $M=$ $g^{2}(p+q-1)-g(p+q)+1$. For all the three backoff schemes, the throughput is given by $\pi_{s}$ and the energy efficiency by $\left(\frac{\pi_{s}}{\pi_{0}+\pi_{f}}\right)$ normalized by the fade margin $(F)$ of the link. We define here one energy unit as corresponding to the transmission of a LL packet with a fade margin of $0 \mathrm{~dB}$. For ideal SR with no backoff, energy efficiency is given by $(1-\epsilon) / F$. Note that maximum theoretical energy efficiency is achieved when there are no failed packets at all, i.e., when $\pi_{f}=0$ which gives $1 / F$ as the theoretical upper bound on the achievable energy efficiency.

For the first-order Markov representation of Rayleigh fading, the relation between average packet error rate $(\epsilon)$, fade margin $(F)$, and parameters $p$ and $q$ are given by [5]: $\epsilon=1-e^{-1 / F}=(1-p) /(2-p-q)$ and $(1-q)=[Q(\theta, \rho \theta)-Q(\rho \theta, \theta)] /\left(e^{1 / F}-1\right)$, where $\theta=\sqrt{2 / F\left(1-\rho^{2}\right)}, \rho=J_{0}\left(2 \pi f_{d} T\right)$ is the correlation coefficient of two samples of the complex amplitude of the Rayleigh fading process ${ }^{1}$ taken $T$ seconds apart, $J_{0}(\cdot)$ is the Bessel function of the first kind and zeroth order, and $Q(\cdot, \cdot)$ is the Marcum $Q$ function.

\section{Results}

We computed the throughput and energy efficiency for all three backoff schemes using the channel parameters $p$ and $q$ for different fade margins and a fixed normalized Doppler bandwidth $f_{d} T=0.001$. The same was found using computer simulation as well. The parameter $g$ for the GBO is chosen to be 0.8 . At $900 \mathrm{MHz}$ carrier frequency, $f_{d} T=0.001$ corresponds to user speed of $1.2 \mathrm{Km} / \mathrm{h}$, link speed of $1 \mathrm{Mbps}$ and LL packet size of 1000 bits. The plots in Figure 2 shows close agreement between analytical and simulation results. Figure 2 and 3 show that the LBO and GBO schemes show good improvement in terms of energy efficiency without noticeable fall in the throughput compared to the ideal SR scheme without backoff. At a throughput of 0.4 , LBO gives an improvement of $3.4 \mathrm{~dB}$ of energy savings while $\mathrm{GBO}$ gives nearly $2.8 \mathrm{~dB}$. Among all the three schemes, LBO is seen to perform best, achieving energy efficiency close to the theoretical upper bound. The BEBO scheme is seen to perform poorer. This is due to the rapid growth of exponentiation in backoff delay, which misses possible successful transmission attempts during good state of the channel. This is bound to improve if we use a truncated backoff, in which the idle length should grow only till a maximum value. Such improvement is evident from Figure 4 which shows the truncated

\footnotetext{
${ }^{1} f_{d}$ is the Doppler bandwidth given by $v / \lambda$, where $v$ is the user velocity and $\lambda$ is the carrier wavelength [2].
}

BEBO performance for different values of $m$. The performance analysis of the LL backoff schemes when used along with transport protocols like TCP and UDP could be an useful extension to this work.

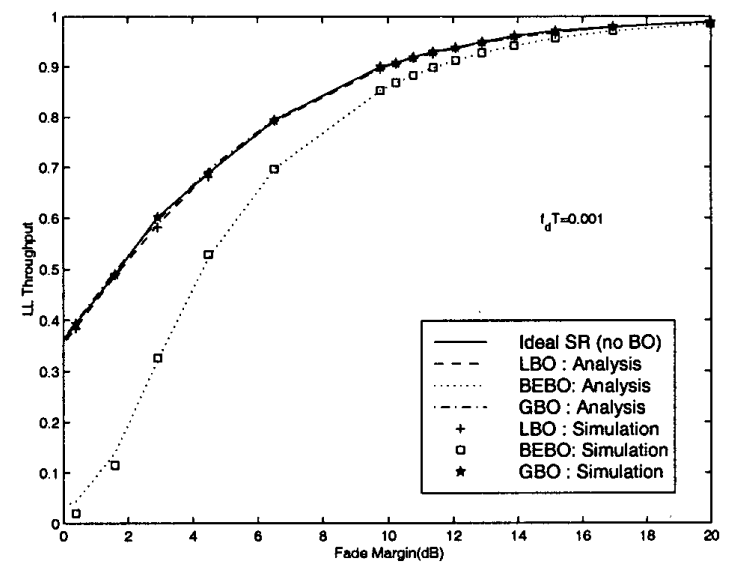

Figure 2: LL throughput $v s$ fade margin performance of LL backoff schemes at $f_{d} T=0.001$.

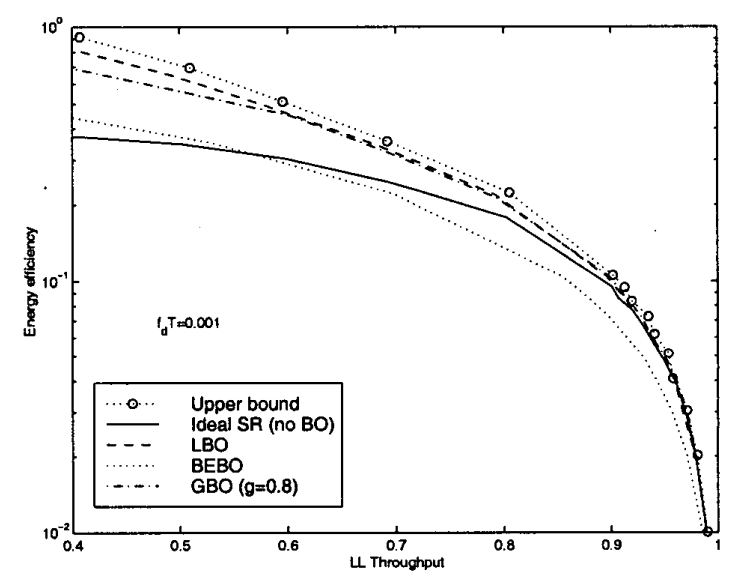

Figure 3: Energy efficiency vs LL throughput performance of LL backoff schemes at $f_{d} T=0.001$.

\section{References}

[1] D. Bertsekas and R. Gallager, Data Networks, PrenticeHall, Englewood Cliffs, New Jersy, 1987.

[2] W. C. Jakes, Jr., Microwave Mobile Communications, New York: John-Wiley \& Sons, 1974.

[3] M. Zorzi and R. R. Rao, "Energy constrained error control for wireless channels," IEEE Personal Communications, vol. 4, pp. 27-33, December 1997.

[4]. H. S. Wang and P. C. Chang, "On verifying the firstorder Markovian assumption for a Rayleigh fading channel model," IEEE Trans. Vehicular Tech., vol 45, no. 2, pp. 353-357, May 1996. 


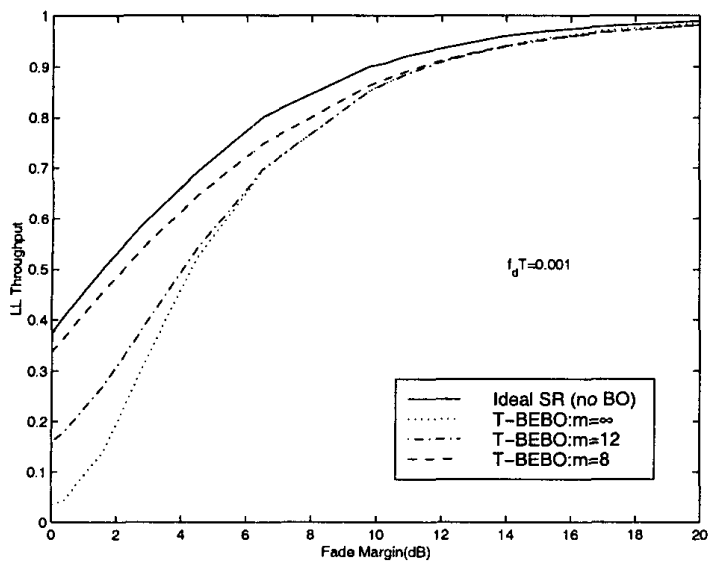

Figure 4: Performance of truncated BEBO for different values of $m$ at $f_{d} T=0.001$.

[5] M. Zorzi, R. R. Rao, and L. B. Milstein, "On the accuracy of a first-order Markov model for data transmission on fading channels," Proc. IEEE ICUPC'95, Tokyo, November 1995.

[6] C. H. C. Leung, Y. Kikumoto, and S. A. Sorensen, "The throughput efficiency of the Go-Back-N ARQ scheme under Markov and related error structures," IEEE Trans. Commun., vol. COM-36, pp. 231-234, February 1988.

[7] L. F Chang, "Throughput estimation of ARQ protocols for a Rayleigh fading channel using fade- and interfadeduration statistics," IEEE Trans. Veh. Tech., vol. VT-40, pp. 223-229, February 1991.

[8] D.-L. Lu and J. F. Chang, "Performance of ARQ protocols in nonindependent channel errors," IEEE Trans. Commun., Vol. 41, pp. 721-730, No. 5, May 1993.

[9] Y. J. Cho and C. K. Un, "Performance of ARQ error controls under Markovian error pattern," IEEE Trans. Commun., vol. COM-42, pp. 2051-2061, February/March/April, 1994.

[10] M. Zorzi and R. R. Rao, "Throughput analysis of ARQ Selective-Repeat protocol with time diversity in Markov channels," Proc. IEEE GLOBECOM'95, November 1995.

[11] R. W. Wolff, Stochastic Modeling and the Theory of Queues, Prentice Hall, 1989. 